\title{
Semi-kinematic mount of the FIREBALL large optics
}

C. Rossin, R. Grange, B. Milliard, L. Martin, G. Moreaux, et al.

C. Rossin, R. Grange, B. Milliard, L. Martin, G. Moreaux, P. Blanchard, J.M. Deharveng, J. Evrard, C. Martin, R. McLean, D. Schiminovich, "Semikinematic mount of the FIREBALL large optics," Proc. SPIE 7018, Advanced Optical and Mechanical Technologies in Telescopes and Instrumentation, 70181Q (23 July 2008); doi: 10.1117/12.788917

EDent: SPIE Astronomical Telescopes + Instrumentation, 2008, Marseille, France 


\title{
Semi-kinematic mount of the FIREBALL large optics
}

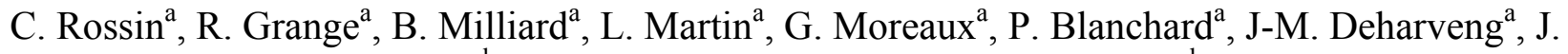

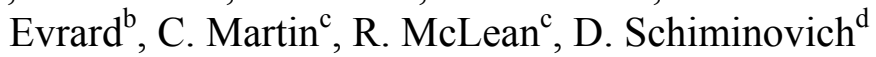 \\ a'Lab. d'Astrophysique de Marseille, UMR 6110 CNRS/Université de Provence, Pôle de l'Etoile site \\ de Château-Gombert, 38 rue Fréderic Joliot-Curie, 13388 Marseille cedex 13 \\ ${ }^{\mathrm{b}}$ CNES Centre Spatial de Toulouse, 18 avenue Edouard Belin, 31401 Toulouse cedex 9 \\ ${ }^{\mathrm{c}}$ California Institute of Technology, 1200 East California Boulevard, Pasadena, CA, USA 91125 \\ ${ }^{\mathrm{d} C}$ Columbia University, 2960 Broadway, New York, NY, USA 10027-6902
}

\begin{abstract}
In the context of the NASA CNES FIREBALL balloon borne experiment, we present the design of a semi-kinematic mount to hold the 1 meter class mirrors of this mission. To maintain these large optics in a reasonable mass and price budgets we choose thin ULE mirrors with a thickness over diameter ratio of 1/16. Such thin mirrors require a multi support mount to reduce self weight deflection. Classical multi support mount used for ground based telescope would not survive the level of shock observed in a balloon experiment either at parachute opening or landing. To firmly maintain these mirrors in several points without noticeably deforming them we investigated the design of a two stages semikinematic mount composed of 24 monopods. We present the detailed design of this innovative mirror mount, the finite element modeling with the deduced optical wavefront deformation. During the FIREBALL integration and flight campaign in July 2007 at CSBF, we confirmed the validity of the mechanical concept by obtaining an image quality well within the required specifications. Variants of this approach are potentially applicable to large thin mirrors on groundbased observatories.
\end{abstract}

Keywords: Balloon experiment, semi-kinematic mount, thin mirrors, shock, monopods, Finite Element Modeling, optical wavefront deformation

\section{INTRODUCTION}

The FIREBALL balloon program (PI Chris Martin) has been funded by NASA and CNES in 2006 to detect and map for the first time the UV emission from the warm hot intergalactic gas (WHIM), which may hide nearly half the total baryons in the local universe. Physical processes in this hot gas are believed to play a critical role in galaxy evolution by their effect on the star formation mechanisms. FIREBALL carries a 1-meter telescope feeding a high throughput image fiber slicing spectrograph to an altitude of $40 \mathrm{~km}$, where observations in the 200-220 nm window can be made with a good transmission despite some residual atmospheric ozone and molecular oxygen.

As for the mechanics, the FIREBALL experiment includes three major subcomponents: the telescope, the spectrograph, and the gondola.

The gondola is the mechanical structure that allows orientation and guidance. It also holds the optical bench and the tracking framework with its associated electronics. The gondola structure which will be described elsewhere is designed as a sparse set of nodes to make a quasi-kinematic structure. Two extra bars are added at a critical point to allow one bar to break without fall down of the whole structure, while introducing no ill constraint. Nodal points are connected with carbon fiber rods; this structure is both stiff and thermally stable (see figure 1).

The telescope consists of a 1.2 meter plano siderostat, situated on a dual axis pivot assembly, feeding a fixed 1.0 meter $\mathrm{f} / 2.5$ paraboloid mirror. The siderostat (see figure 2) provides elevation control of a boresite, over an elevation range of 45-70 degrees, as well as fine cross-elevation control. Coarse azimuth orientation is provided by the gondola pivot mechanism.

Advanced Optical and Mechanical Technologies in Telescopes and Instrumentation, edited by Eli Atad-Ettedgui, Dietrich Lemke, Proc. of SPIE Vol. 7018, 70181Q, (2008) · 0277-786X/08/\$18 · doi: 10.1117/12.788917 
The spectrograph is a Littrow Offner design, with a spherical mirror (diameter $480 \mathrm{~mm}$ ) and concentric convex spherical grating. The optical interface through a flexible fiber bundle allows a clean interface with no alignment constraint with respect to the telescope.

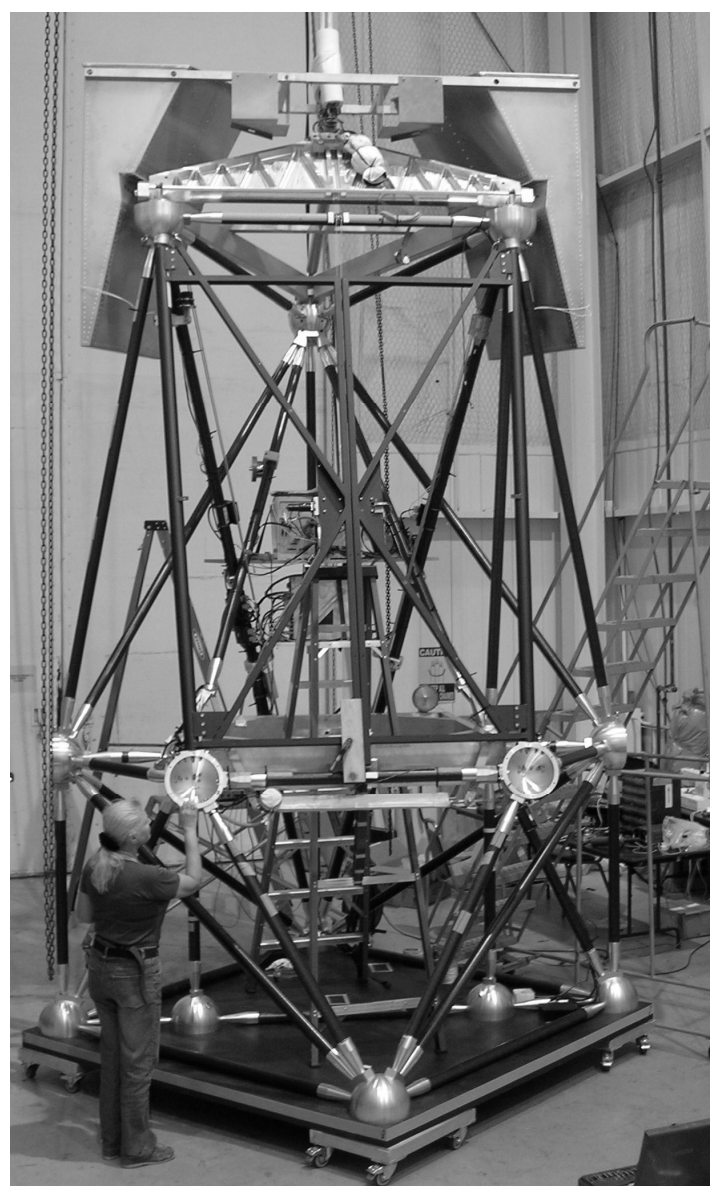

Figure 1: Structure of the gondola

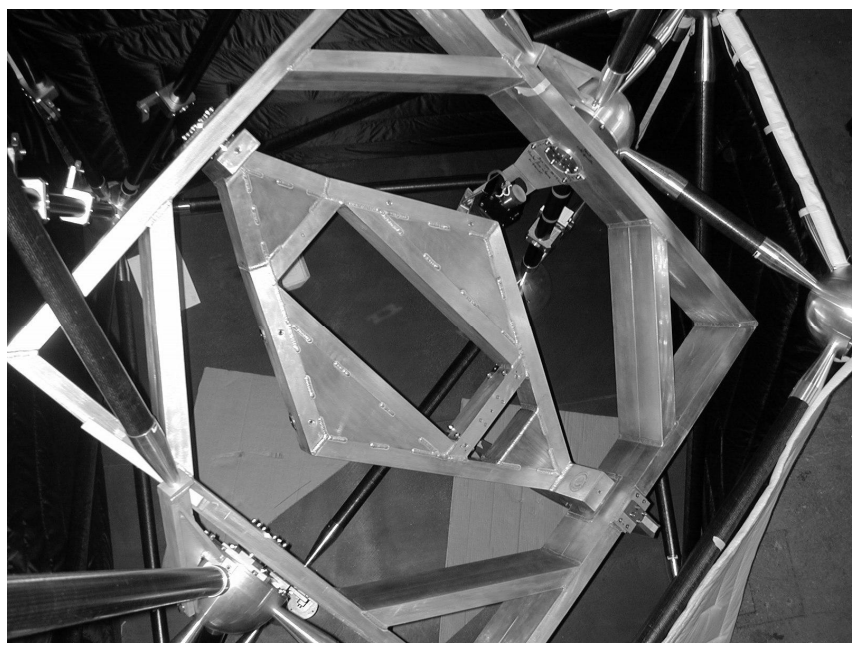

Figure 2: Photo of the siderostat mechanical structure

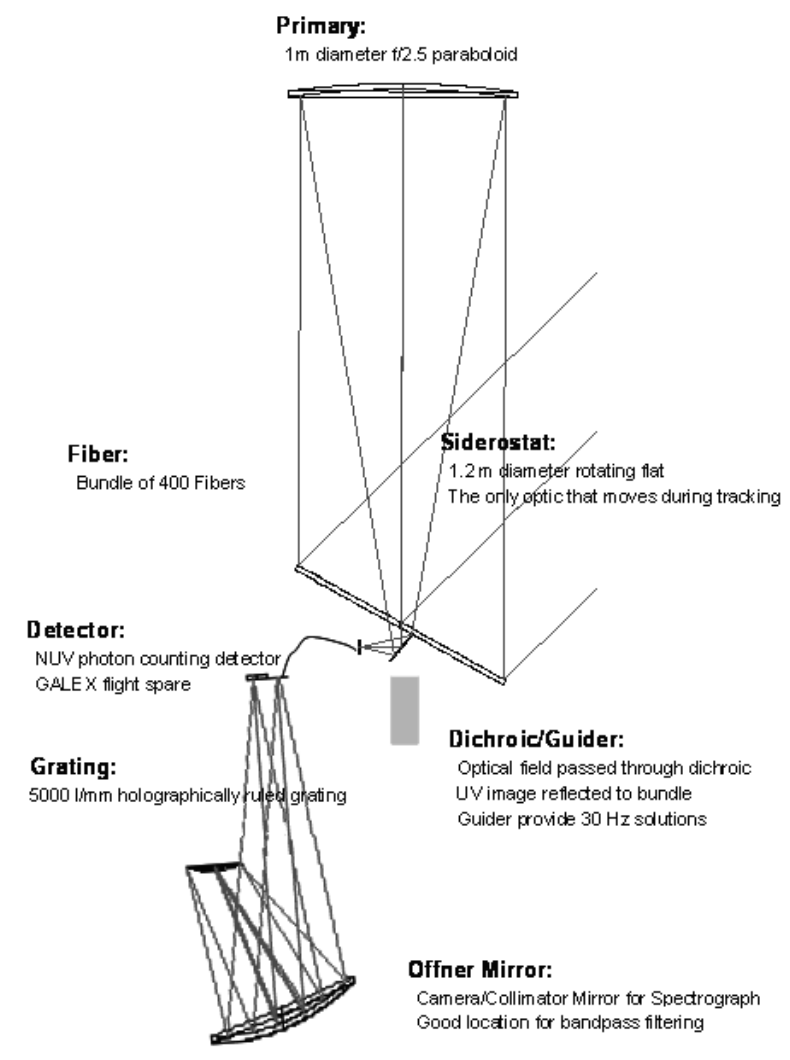

Figure 3: Optical layout

The optical layout of FIREBALL is shown in figure 3. Incoming light strikes the siderostat, the primary mirror (paraboloid), the dichroic, beam splitter, enters the UV fiber, exits the fiber and enters the Offner Spectrograph, strikes the Offner sphere, holographic grating, and sphere again, finally striking the UV detector. 


\section{LARGE OPTICS MOUNTS SPECIFICATION}

\subsection{Optical specifications}

To achieve the FIREBALL spatial resolution of 10 arcsec the allocated budget to both large optics is that $80 \%$ of the encircled energy is within 3 arcsec. This budget has to be split into manufacturing errors and deshape of the mount due to self deflection under gravity and thermal load. We eventually found that a 9-points support configuration would limit the wavefront deshape contribution to less than 1 arcsecond to the image size.

The large mirrors were fabricated from low thermal expansion ULE Corning Code 7972 blanks by Optical Mechanics. We show a photo of the mirrors at the optical shop in figure 4. The Al-MgF2 mirrors coating is optimized for the UV.

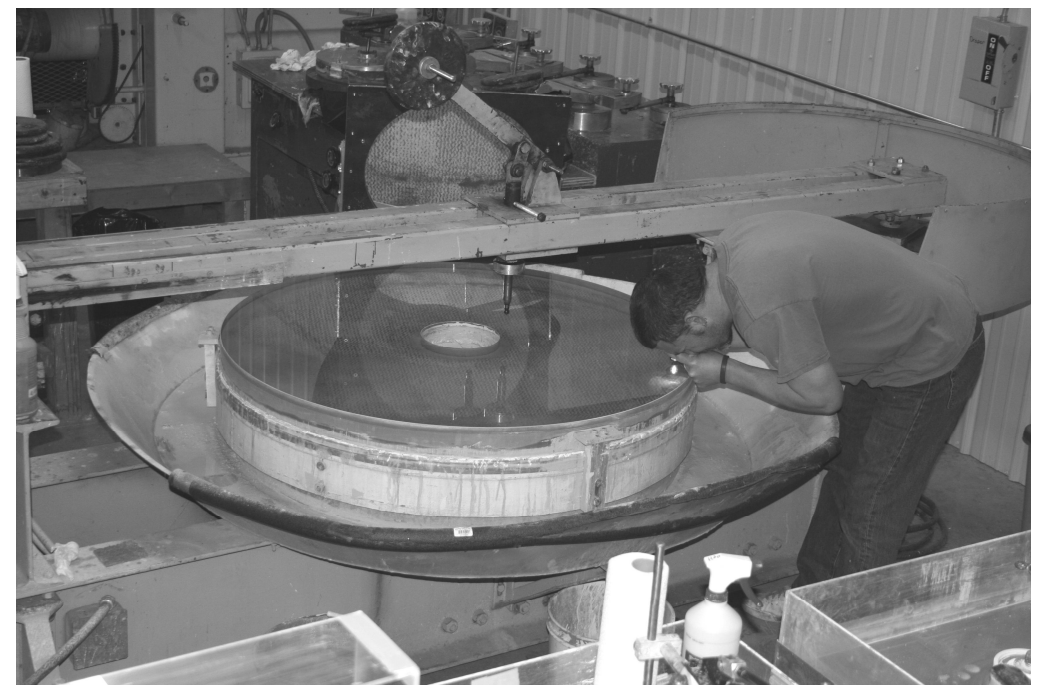

Figure 4: FIREBALL 1.2m diameter Siderostat mirror undergoing testing at Optical Mechanics, Inc.

\subsection{Mechanical specifications}

The design of the mount must maintain the optical performances of the large optics under the balloon environment. This is translated into two major specifications in terms of thermo-elastic and dynamic behavior.

\subsubsection{Thermo-elastic behavior: gondola design load}

The effect on the image of the self-weight deshape (gravity vector vertical and 20 deg tilted) of the two mirror telescope should be less than 1 arcsec (maximum difference between any points) for a temperature range of $+20^{\circ} \mathrm{C}$ to $-20^{\circ} \mathrm{C}$.

Under the specified $40^{\circ} \mathrm{C}$ thermal change, the radius of curvature of both large optics could vary due to the contraction of the aluminum supporting frame. This additional curvature should be less than $10^{-5} \mathrm{~m}$.

\subsubsection{Dynamic behavior}

The first mode of large optics shall be above $17 \mathrm{~Hz}$ to be outside the band pass of the gondola pointing loop.

For the siderostat, this frequency corresponds to a configuration with this latter mounted on both cross-elevation and elevation frames, with boundary conditions as in operation. That means the cross elevation frame can rotate and slide inside the elevation frame, which also can rotate and slide inside the gondola.

The gondola equipments shall also be able to sustain to an equivalent static shock (parachute opening) of $15 \mathrm{~g}$ vertical load, $7.5 \mathrm{~g}$ load at 45 degrees from the vertical and $7.5 \mathrm{~g}$ horizontal load without failure (CSBF requirements).

On top of that, the large optics mount shall withstand the landing of the gondola. Thus, in order to check their real dynamic behavior, we have added a half-sine shock calculation to simulate $10 \mathrm{~g}$ at $30 \mathrm{deg}$ from the vertical (-Z direction) (CNES recommendation). 


\section{MOUNT DESIGN PHILOSOPHY}

\subsection{Multi supported thin mirrors}

To minimize the self weight deshape under gravity, the first approach was based on a double arch blank maintained on three or six points. It appeared rapidly that this type of blank shape was largely outside the budget cap for our balloon experiment.

To save cost and weight on the large mirrors, we decided to rely on a thin mirror concept (diameter to thickness ratio 16/1) that in contrast requires a strong engineering effort in the mount design to maintain the optical performances.

Thin mirrors have to be supported by several points to minimize the self weight deshape. Using a free software crosschecked with Nastran (PLOP software at http://www.davidlewistoronto.com/plop/), we finally decided to use a 9 points support in two rings as a good compromise between total deformation, stiffness and complexity.

For a ground based experiment it's classical to use whiffle tree support or Hindle mount: each set of three support points is connected by a delta plate pivoting on a spherical ball at its center of gravity and each pair of delta plate is being linked by a bar using universal joint. When the mirror is used at variable orientation it is required to add an appropriate radial support which could use counter-weighted levers to vary the forces acting on the mirror edge as a function of the changing direction of the gravity.

\subsection{Semi-kinematic mounts with hexapod}

In contrast with ground based experiment, the FIREBALL mirrors have to be maintained firmly to withstand severe shock specifications and thermal environment. Keeping the idea of the whiffle tree support, and since both large optics are made from ULE silica, a brittle material, we studied a mount design to isolate as much as possible the optical element from mechanical and thermal strains.

Our design approach is to link the 9 points inside the mirror (corresponding to 18 points on the rear surface of the optics) to the cross elevation frame (for the siderostat) or the parabolic frame by means of two stages of hexapods. These hexapods which are semi-kinematic mounts allow handling the mirror at its center of gravity (with no additional torques) even if the mount is not directly connected to it.

The mirror mount is made of two stages: three hexapods link the mirror to three boxes (first stage) and these three boxes are linked to the frame by a single hexapod (second stage). The intersection point of the monopods of the first level, which correspond to the instantaneous pivot, was designed to be in the plane containing the center of gravity of the mirror. The intersection point of the monopods of the second level was designed to be in the plane containing the center of gravity of the mirror plus the boxes equipped with their hexapods (see fig. 5 below). In the bipod design we adjusted the angle between legs to have the same strength in the three axes.

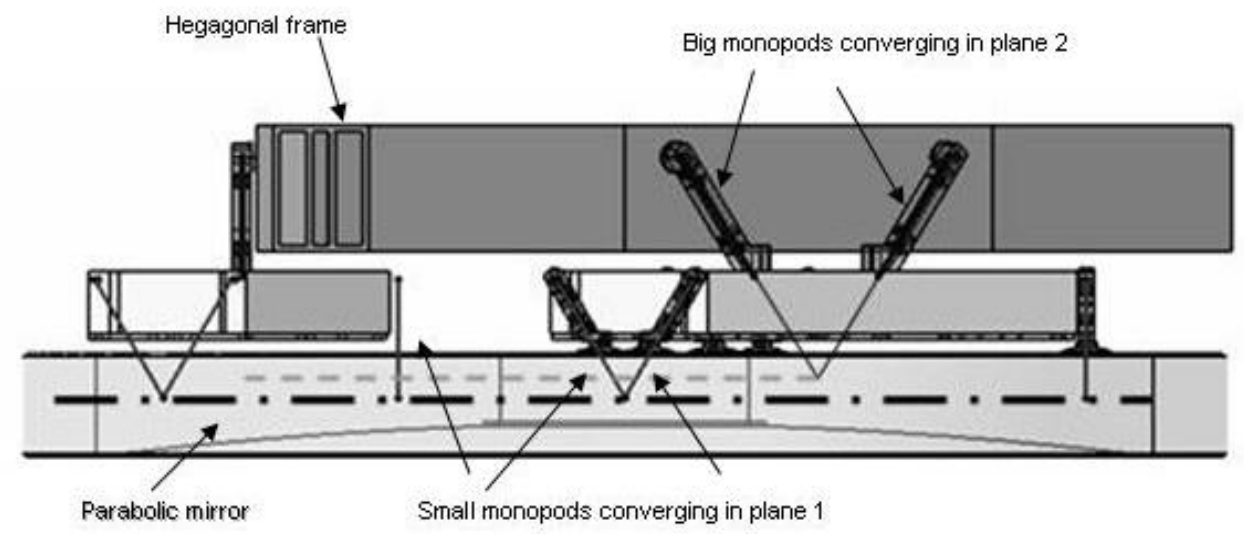

Figure 5: Parabolic mirror mount with both stages of hexapods (cut-view)

For monopods manufacturing, we chose titanium primarily because of its high yield strength and low Young modulus. Its resistance to corrosion, medium density and nonmagnetic property are added interested features. 
We bonded the rear face of the optics to 18 Invar pads fixed at the bottom parts of the monopods with a 100 micrometers layer of 3M 2216 epoxy.

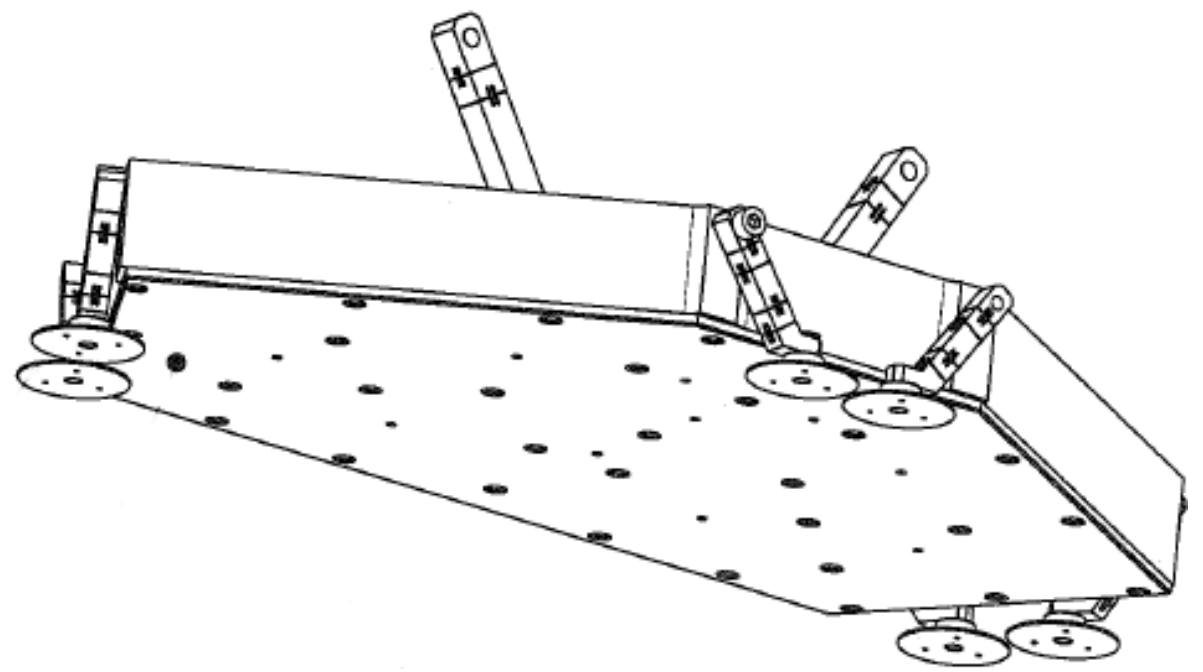

Figure 6: Design of a suspension box equipped with one hexapod which will be connected on the rear face of the mirror

\subsection{Flexures design}

The ends of each monopods leg have to be rotationally compliant. Among the different possible pivot flexures, hinges ([1], [2] and [3]) rapidly appeared to us as a clever solution to provide both the required stiffness to maintain alignment and the compliance to avoid optics deformation from the mount. The compliance of a flexure hinge is free of play, stickslip and friction effects, in contrast with spherical ball flexures.

The two axis flexure at each end of the legs consists of two single axis flexures at right angles to each other (see Fig.7 and Fig. 8) equipped with a limit stop with maximal range of $0.3 \mathrm{~mm}$ to prevent from exceeding the yield strength of the material in case of mishandling during the integration process.

The design of the flexures of FIREBALL monopods is a compromise between a great compliance of the flexure and high resistance to shock loads (tension, compression and buckling). Their contribution to the decrease of the first frequency has also to be taken into account in the design. To know the forces in the monopods and the first mode, a simplified model of the hinges has been used, considering them as ideal flexures (rod elements).

To calculate the compliance with the required stiffness, Paros and Weisbord ${ }^{[4]}$ equations have been used and validated comparing the results with $3 \mathrm{D}$ finite element analysis. This work has shown that the equations are reliable with a maximal error of $15 \%$. These calculated compliances have been used in analytical models to determinate the torques and forces on the monopods when they deshape under stress due to machining default, alignment errors and thermo-elastic load case.

These additional torques and forces have been added to the ideal flexures in the Finite Element Model in order to analyze the resulting stress and deshape on the large optics. 


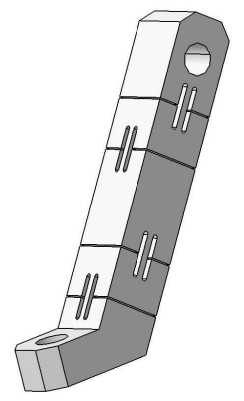

Figure 7: geometry of the small monopods

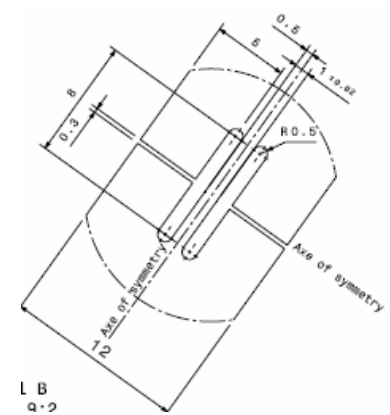

Figure 8: detailed geometry of one hinge

\section{FINITE ELEMENT MODELING}

This analysis had been carried out using MSC/NASTRAN software.

Two models have been designed and analyzed:

- the first composed of the siderostat mirror, the mounts (monopods and boxes), the cross elevation frame and the elevation frame,

- and the second composed of the parabolic mirror, the mounts (monopods and boxes), the parabolic frame and the rods linking parabolic frame to the gondola spheres.

\subsection{Modal analysis}

4.1.1 Modal analysis of the Siderostat mount

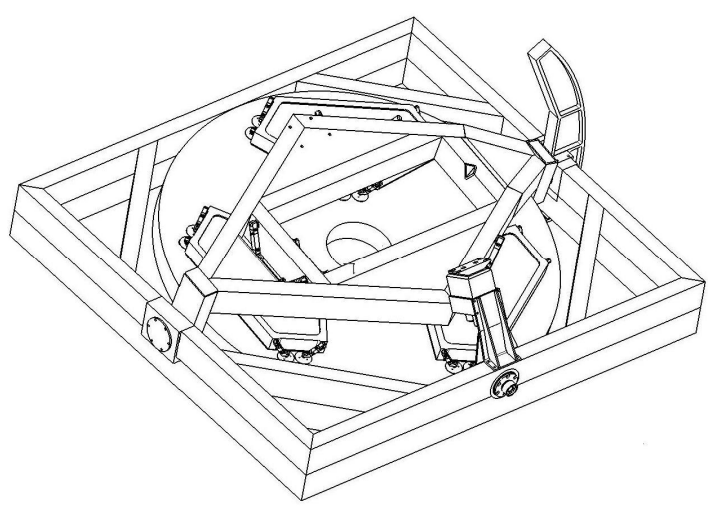

Figure 9: CAD design of the siderostat mount

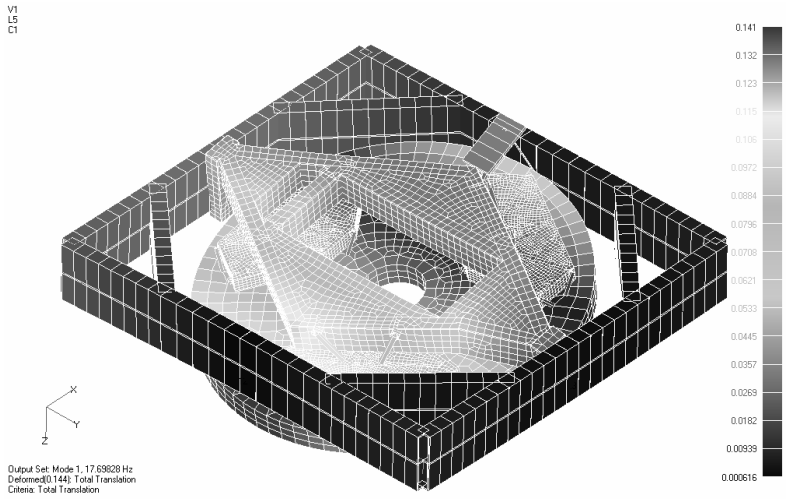

Figure 10: First vibration mode of the siderostat mount

The modal analysis of the siderostat mirror mount led us to the following results and mode shape description:

$\mathrm{F} 1=17.7 \mathrm{~Hz} \Rightarrow$ Global rotation mode of the siderostat mirror mount along a line passing through the rotating bearings (cf. figure 10),

$\mathrm{F} 2=21.3 \mathrm{~Hz} \Rightarrow$ Global translation mode of the siderostat mirror due to a flexion of a part of the frame,

$\mathrm{F} 3=29.3 \mathrm{~Hz} \Rightarrow$ Global rotation mode of the siderostat mirror mount along a line passing through the ball bearings and due to a global flexion mode of the elevation frame. 


\subsubsection{Modal analysis of the Parabolic mirror}

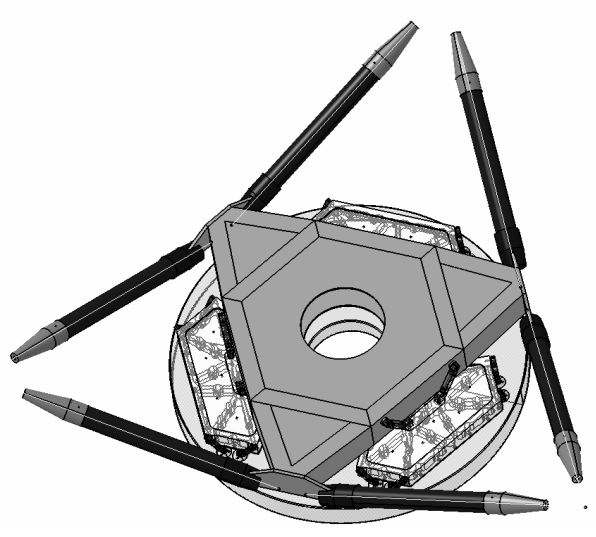

Figure 11: CAD design of the parabolic mirror mount

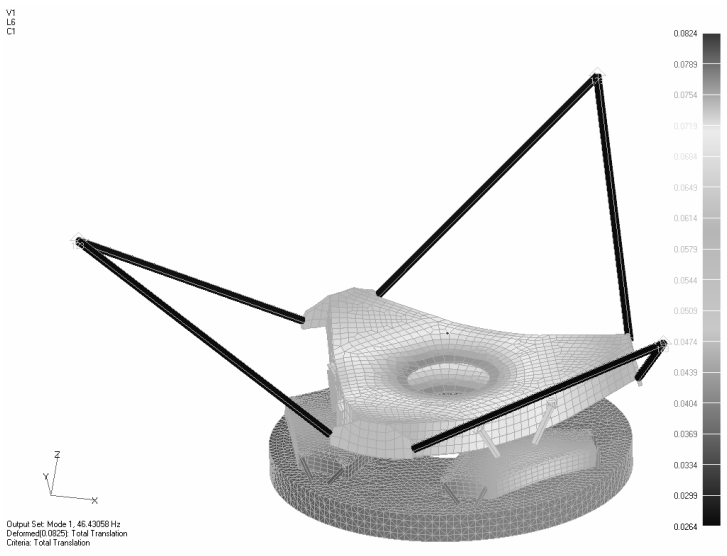

Figure 12: First vibration mode of the parabolic mirror mount

The modal analysis of the parabolic mirror led us to the following results and mode shape description:

$\mathrm{F} 1=46.4 \mathrm{~Hz} \Rightarrow$ Global translation mode of the parabolic mirror mount in the optical axis direction (cf. figure 12),

$\mathrm{F} 2=50.4 \mathrm{~Hz} \Rightarrow$ Global rotation mode of the parabolic mirror along a line passing through the frame,

$\mathrm{F} 3=50.4 \mathrm{~Hz} \Rightarrow$ Global rotation mode of the parabolic mirror mount along a line perpendicular to the previous one, symmetrical mode to the second one.

\subsection{Static and dynamic analysis for large optics}

For both models (parabolic and siderostat), we have verified static deformation and stresses on the mirror, the boxes and the supporting frame. We have also checked the tension and compression forces, plus the buckling forces encountered in all monopods of both stages between the mirror and the supporting frame (see $\S 4.3$ ).

The dynamic analysis is a transient response to a half-sine shock (defined in chapter 2.2.4). It has been applied directly on the interface nodes of the large optics support mounts with the gondola. It has been applied at 30 degrees in $-Z$ direction with an angle theta of 150 degrees in XY plane (calculated to be the worst case).

All FEA results (for static and dynamic analysis) are not shown here, but the values of the maximum Von Mises stresses in all components of the large optics mounts were below the design allowable strength value of the different materials.

The following figures present some results for some of the siderostat parts under static and dynamic load case.

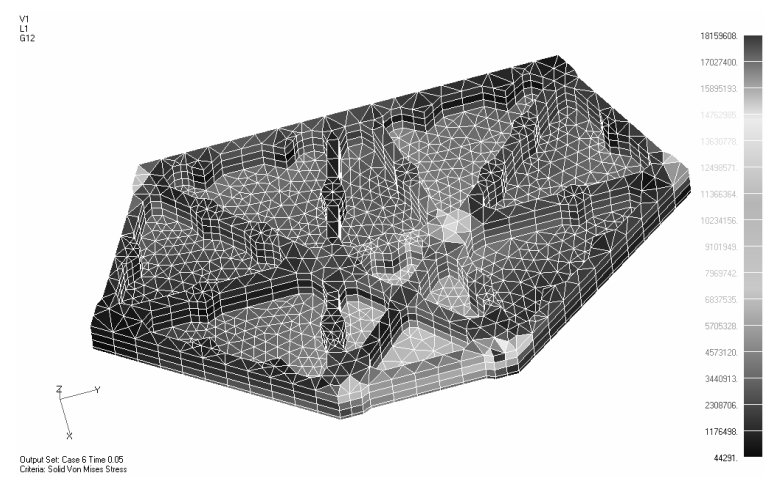

Figure 13: Box (shown opened) under half-sine shock

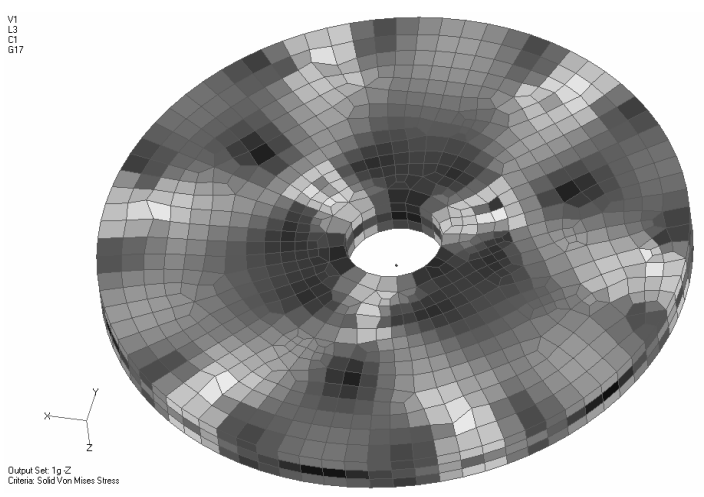

Figure 14: Siderostat stresses under $1 \mathrm{~g}$ in $-\mathrm{Z}$ direction 


\subsection{Detailed analysis of the monopods}

As the finite element model analyses have been made with simplified elements for the monopods (rod elements), some detailed analysis have been done in order to check that the hinges of big monopods (hexapod between the boxes and the frame) and small monopods (hexapod between the mirror and the boxes) will stand the axial forces due to the different load cases.

Stress analysis, due to static and half-sine shock load cases have been done for both models (parabolic and siderostat).

Following tables summarize the values of forces generated in the monopods, the stresses calculated in the hinges and the margins of safety compared to the ultimate strength of titanium used (974 MPa), as the requirement is not to break at landing.

\begin{tabular}{|l|c|c|c|}
\cline { 2 - 4 } \multicolumn{1}{c|}{} & $\begin{array}{c}\text { Max. rod axial force } \\
\text { due to worst static load case }\end{array}$ & Stress in the hinges & MOSu \\
\hline Siderostat small monopods & $-6308 \mathrm{~N}$ & $404 \mathrm{MPa}$ & 2.4 \\
\hline Siderostat big monopods & $-9903 \mathrm{~N}$ & $344 \mathrm{MPa}$ & 2.8 \\
\hline Parabolic small monopods & $4254 \mathrm{~N}$ & $354 \mathrm{MPa}$ & 2.7 \\
\hline Parabolic big monopods & $6908 \mathrm{~N}$ & $240 \mathrm{MPa}$ & 4.0 \\
\hline
\end{tabular}

Table 1: Margin of safety in all siderostat and parabolic monopods for static load cases

\begin{tabular}{|l|c|c|c|}
\cline { 2 - 4 } \multicolumn{1}{c|}{} & $\begin{array}{c}\text { Max. rod axial force } \\
\text { due to the half-sine shock }\end{array}$ & $\begin{array}{c}\text { Stress value in } \\
\text { the hinges }\end{array}$ & MOSu \\
\hline Siderostat small monopods & $-5376 \mathrm{~N}$ & $345 \mathrm{MPa}$ & 2.8 \\
\hline Siderostat big monopods & $-10186 \mathrm{~N}$ & $354 \mathrm{MPa}$ & 2.7 \\
\hline Parabolic small monopods & $4177 \mathrm{~N}$ & $348 \mathrm{MPa}$ & 2.8 \\
\hline Parabolic big monopods & $6891 \mathrm{~N}$ & $239 \mathrm{MPa}$ & 4.0 \\
\hline
\end{tabular}

Table 2: Margin of safety in all monopods for half-sine shock

On top of that, considering the high value of compression exerted on the small and big monopods in a few load cases, a buckling analysis has been made on the hinges alone. The analysis has shown that these high compression values did not exceed the critical buckling loads for the hinges.

However, another buckling analysis has also been made on the entire small monopods (the one with the thinnest hinges) for siderostat and parabolic mirrors. Here below is a figure showing the deformation mode of the buckling of one monopod (of the siderostat). As the buckling force limit $(27700 \mathrm{~N})$ for the whole monopod is well above the maximum rod compression force of this small monopod $(6308 \mathrm{~N})$, there is no risk of buckling of the whole monopod. 


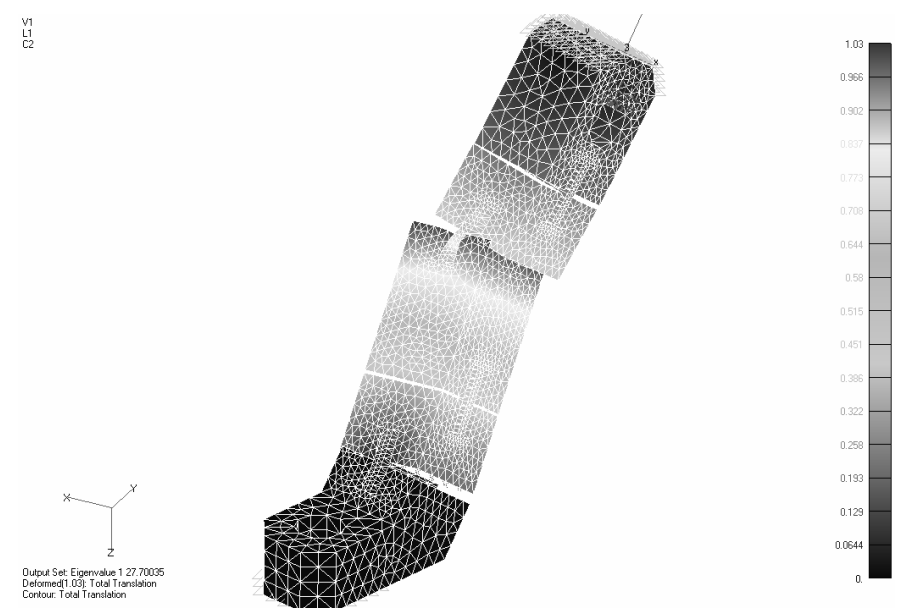

Figure 15: Buckling deformation of a small monopod fixed at the bottom and guided at the top part

\section{OPTICAL WAVERONT DEFORMATION FOR THE SIDEROSTAT}

\subsection{Thermo-elastic analysis}

A load corresponding to a thermal decrease of $40^{\circ} \mathrm{C}$ of the siderostat mount has been simulated. The thermal effect has been associated with gravity loading in order to look at the deformed shape of the mirror in operation and then with gravity at 20 deg around $\mathrm{X}$ direction to take into account the fact that the siderostat mirror can rotate around X.

NASTRAN results have been translated to Zemax (optical software) in order to check the aberrations of the siderostat for the worst load case associating thermal effect and gravity at $20 \mathrm{deg}$. We made the assumption that the main $\mathrm{Al}$ frame and the intermediate aluminum boxes are at $-20^{\circ} \mathrm{C}$.

\subsection{Optical distortion induced by thermal deformation of the mount combined with gravity}

Results of the combined load case of gravity added to thermal deformation for the siderostat were given as a file with coordinates of the different nodes of the active surface and their displacements in $\mathrm{Z}$ direction (orthogonal to the surface). Then these figures were processed through Zemax software in order to obtain a surface sag map (see figure 16) and a spot diagram (figure 17).

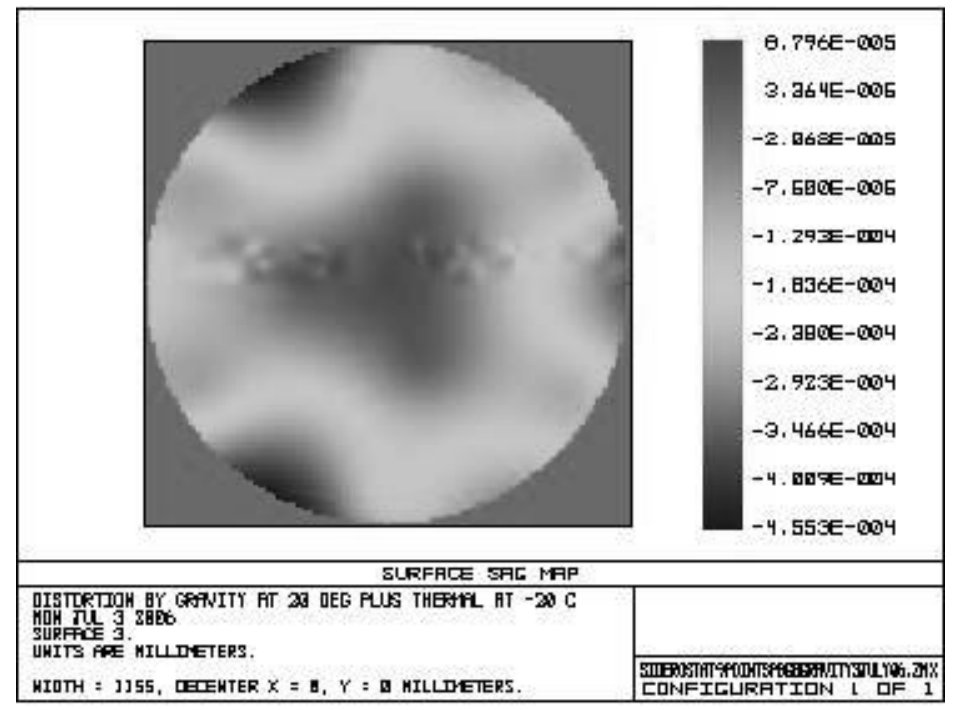

Figure 16: Surface sag map induced by gravity added to thermal deformation for the siderostat mirror 


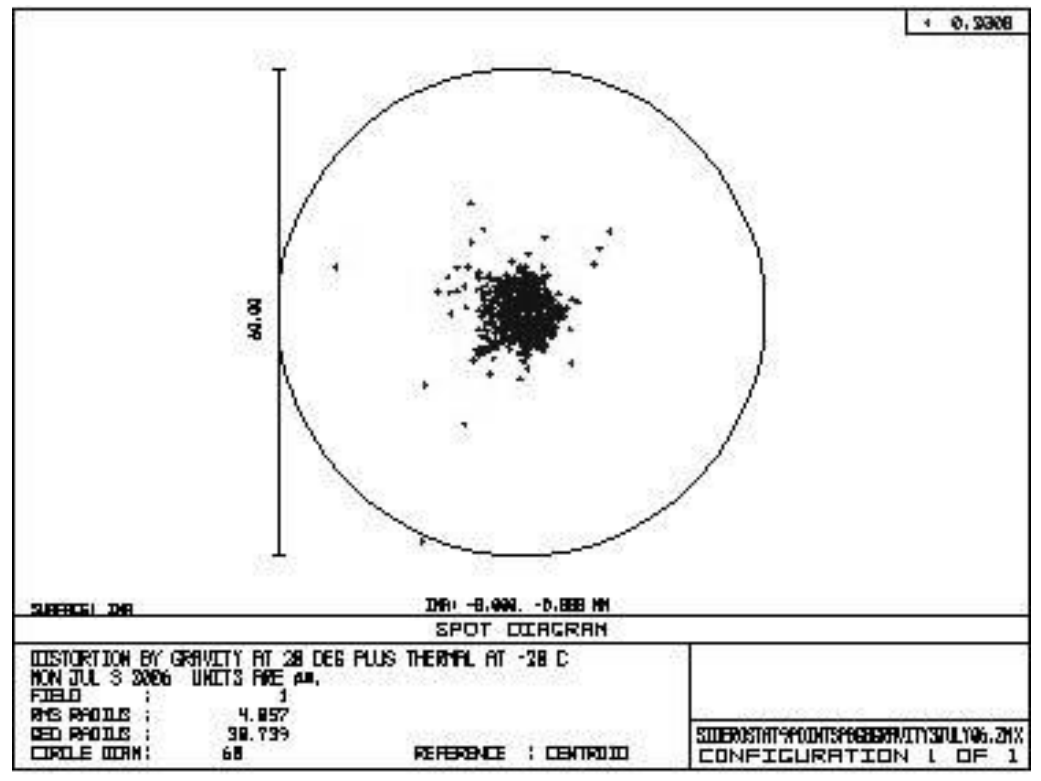

Figure 17: Spot diagram induced by gravity added to thermal deformation for the siderostat mirror

The conclusion of this study is that the effects of the thermal deformation combined with gravity on the optical distortion of the large optics have been minimized thanks to this mount. $80 \%$ of the encircled energy is in a 9 microns circle or 0.75 arcsecond. The defocus of the siderostat is of 17 microns only, which is acceptable.

In addition, even with a large differential thermal effect between the ULE mirrors and the aluminum frames, the optics are kept well centered on the telescope axis thanks to the flexures of each monopods.

\section{INTEGRATION AND TESTS PHASES}

AIT/AIV phases took place in Columbia Scientific Balloon Facility during, May, June and July 2007 with the active participation of different teams (CNES, LAM, Caltech and Columbia University).

The picture below shows one of the phases of the integration.

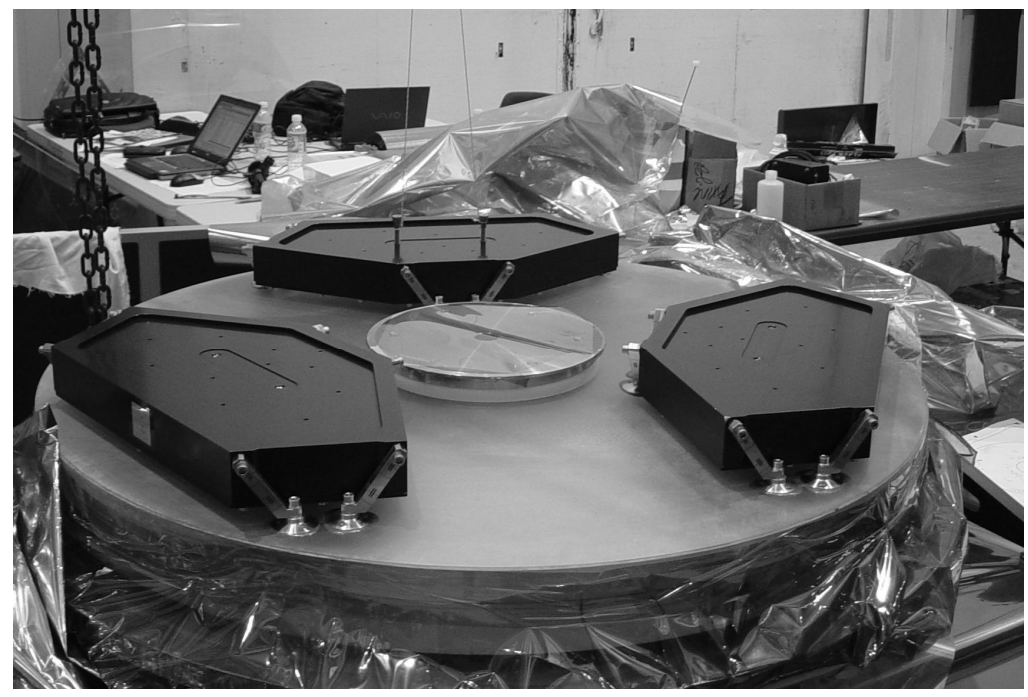

Figure 18: Integration of the boxes on the parabolic mirror

Then we proceeded to some tests and repetition before the launch took place on $22^{\text {nd }}$ of July 2007. 


\section{CONCLUSIONS AND FUTURE WORK}

Stiffness, static and dynamic behavior (due to landing of the gondola) and thermo-elastic analysis did confirm that the design of the large optics mirror mounts meet the global requirements. In addition, translation of the displacements results of the optical surface of both mirrors to Zemax (optical software) has demonstrated optical aberrations well within specifications.

Therefore, we can say that all calculations presented in this document show that the design of the mirror mounts maintains the optical performances of the large optics under the balloon environment.

On top of that, we confirmed the validity of the mechanical concept by obtaining an image quality well within the required specifications, during the FIREBALL integration campaign in July 2007 at CSBF.

\section{ACKNOWLEDGMENTS}

The authors would like to acknowledge all people from the Columbia Scientific Balloon Facility, which has provided complete balloon operations services and engineering support to FIREBALL project, and Jean-Lucien Boit (LAM) for many helpful comments.

Special thanks also go to CNES, Caltech and Columbia University teams for their work and constant support during integration and tests phases.

\section{REFERENCES}

[1] Chin, D., "Optical Mirror-Mount Design and Philosophy”, Applied Optics, Vol. 3, 895-901 (1964).

[2] Hog, E., "A Kinematic Mounting", Astronomy \& Astrophysics 41, 107-109 (1975).

[3] Vukobratovich, D. and Richard, R. M., "Flexure Mounts for High-Resolution Optical Elements", Proc. SPIE 959, 18-36 (1988).

[4] Paros, J.M. and Weisbord, L., "How to Design Flexure Hinges", Machine Design, 151-156 (1965). 